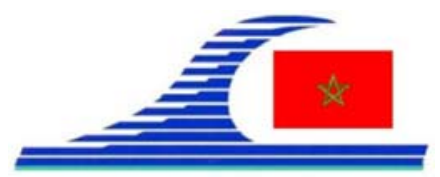

\title{
Impacts du dragage d'exploitation sur les fonds littoraux : cas de Mehdia
}

\section{Aicha BENMOHAMMADI ${ }^{1}$, Mustapha LABRAIMI ${ }^{2}$}

1. Université Ibn Tofail, Faculté des Sciences, Equipe Géosciences de l’Environnement, L. B.E.Q, Kénitra, Maroc. abenmohammadi@gmail.com

2. Université Mohammed V-Agdal, Faculté des Sciences, Geomap, Rabat, Maroc. labraimimustapha@yahoo.fr

\section{Résumé :}

L'exploitation des fonds littoraux par dragage au large de Mehdia (Maroc) impacte le littoral en contribuant à son recul. Le prélèvement de sédiment dans la plage sousmarine et les excavations qui en découlent perturbent gravement la dynamique sédimentaire de la zone littorale et menacent sa stabilité.

\section{Mots-clés :}

Impact - Dragage - Littoral - Mehdia - Maroc

\section{Introduction}

Au Maroc, la demande de plus en plus croissante en sable, en tant que matériau de construction, a obligé les utilisateurs dans le domaine des BTP à rechercher d'autres sources d'approvisionnement pour répondre à la forte demande, particulièrement dans la zone Nord du pays. De l'utilisation du sable de concassage, on est ainsi passé à l'utilisation du sable de dragage des fonds marins; les sables alluvionnaires restant limités dans leur exploitation.

Cette diversification dans l'exploitation de la ressource en sable n'a pas été sans soulever un certain nombre de problèmes aussi bien scientifiques que socioéconomiques et juridiques du fait des impacts de son exploitation sur l'environnement et des exigences d'une saine concurrence dans la commercialisation d'un produit marchand de plus en plus convoité.

Historiquement, le dragage des fonds marins, est en liaison directe avec l'établissement de ports sur le littoral. Il s'effectue dès l'installation des aires portuaires et se continue pour entretenir et/ou approfondir les chenaux et les bassins pour permettre l'accès des navires aux installations portuaires.

De par son impact, CLARK (1977) caractérisait le dragage comme la menace la plus sérieuse pour les écosystèmes littoraux.

Les incidences de l'extraction des granulats des fonds de la zone littorale sur l'environnement ont été mises en évidence à l'échelle mondiale et ont induit la mise en place par les gouvernements d'instruments, nombreux et diversifiés, dans le but de 
La connaissance de la Mer :

un vecteur du développement durable en Méditerranée

permettre une réflexion sur la faisabilité, la rentabilité et la réglementation de l'exploitation du sable au large du littoral. Dans un document d'orientation sur la mise en œuvre des activités extractives non énergétiques conformément aux critères Natura 2000 (COMMISSION EUROPEENNE, 2011) on relève, entre autres, que "l'incidence la plus significative du dragage est l'abaissement du niveau des fonds marins et l'enlèvement des sédiments marins, ce qui entraîne une altération temporaire de la topographie, de la composition et de la structure sédimentaires. En outre, l'extraction du substrat provoque la destruction partielle, voire totale à terme, de la biocénose benthique. Les changements infligés à la morphologie des fonds marins et à la nature des sédiments de surface, ainsi que les effets sur la macrofaune qui y sont liés se révèlent plus profonds et plus durables lorsque les activités d'extraction sont plus intensives, ou lorsque les sites d'extraction se trouvent dans des milieux stables".

Ainsi, les activités de dragage peuvent affecter les processus côtiers de plusieurs manières : (i) par l'enlèvement de matériaux qui protègent la côte contre l'érosion, (ii) en contribuant au déficit sédimentaire de la cellule sédimentaire côtière, (iii) en augmentant la hauteur d'eau, ce qui a pour résultat de modifier à la fois la réfraction des vagues et les caractéristiques de dérive littorale.

\section{Résultats}

Au Maroc, l'extraction des granulats marins est devenue une réalité depuis 1997. On traitera dans ce qui suit l'impact des dragages des fonds littoraux à Mehdia, en se limitant aux conséquences de cette exploitation sur le trait de côte. Le volume de sable commercialisé de septembre 2004 à fin 2009, tel que déclaré par l'exploitant du dragage d'exploitation entrepris à Mehdia s'élève à $1302183 \mathrm{~m}^{3}$, soit 32 fois environ la moyenne du transit littoral annuel orienté du Nord vers le Sud. (figure 1).

La morphologie du littoral nord atlantique du Maroc, du Gharb au Tangérois, est caractérisée par la présence de barres sableuses dont le comportement détermine la configuration du littoral dans son ensemble (EL ABDELLAOUI \& OZER, 2005). Elles forment un stock sableux mobile qui contribue à l'équilibre morphosédimentaire des plages et participe, lors des contextes hydrodynamiques agressifs, à la protection de leurs parties aériennes.

L'estimation de la limite d'action des houles moyennes (KOMAR, 1998) au large du Gharb permet de séparer la plage sous-marine du reste du plateau continental (voir tableau 1).

Les calculs ont été réalisés sur la base de la série temporelle des houles arrivant sur le site. Dans 93.9 \% du temps, la limite d'action des houles moyennes se situe entre $19 \mathrm{~m}$ et $60 \mathrm{~m}$ de profondeur. Cette limite montre l'étendue de la plage sous-marine où s'effectuent les processus sédimentaires qui sont perturbés par le dragage d'exploitation sur presque la totalité de l’année. 
Tableau 1. Limite d'action des houles moyennes au large de Mehdia (Maroc)

\begin{tabular}{|c|c|c|c|c|c|}
\hline \multirow[t]{2}{*}{ Direction } & \multicolumn{4}{|c|}{$\begin{array}{l}\text { Estimation de la limite d'action des houles moyennes, } \\
\text { en } m .\end{array}$} & \multirow{2}{*}{$\begin{array}{l}\text { Occurrence } \\
\text { annuelle } \\
\text { en } \%\end{array}$} \\
\hline & Printemps & Eté & Automne & Hiver & \\
\hline$N$ & 9.37 & 7.08 & 12.68 & 11.80 & 0.45 \\
\hline$N N W$ & 34.48 & 19.84 & 37.41 & 49.84 & 19.58 \\
\hline$N W$ & 44.85 & 28.66 & 46.03 & 60.59 & 52.08 \\
\hline$W N W$ & 46.71 & 30.98 & 44.3 & 60.01 & 22.24 \\
\hline$W$ & 32.11 & 16.03 & 28.66 & 39.89 & 2.84 \\
\hline WSW & 14.14 & 7.97 & 15.63 & 21.08 & 0.75 \\
\hline SW & 9.52 & 5.03 & 12.06 & 12.41 & 0.08 \\
\hline SSW & 10.71 & - & 3.01 & 5.87 & 0.01 \\
\hline
\end{tabular}

D'autre part, selon une étude d'expertise sédimentologique de la plage de Mehdia effectuée par SOGREAH (2011), la cote de fermeture est estimée à -10.5 m ZH (zéro hydrographique) alors que l'exploitant du dragage déclare "on ne creuse pas au-delà de $7 \mathrm{~m}$ de profondeur" (RBOUB, 2011). La même étude conclut que "la réalisation ces dernières années de dragage d'exploitation au niveau du cône alluvial a permis de créer des surprofondeurs au niveau de l'embouchure de l'Oued et donc de mieux piéger les sédiments dans cette zone".

\section{Conclusions}

La tendance érosive de la plage de Mehdia se trouve accélérée par l'action anthropique qui introduit un déséquilibre dans les processus côtiers en limitant le volume de sable nécessaire au maintien des plages et à la stabilité du trait de côte. Le recul est estimé à 4 m/an entre 1997 et 2009. (SOGREAH, 2011). La destruction des barres sableuses, par le prélèvement de sédiments des fonds littoraux, aggrave le déficit sédimentaire de la zone littorale et perturbe la dynamique sédimentaire locale. L'impact du dragage d'exploitation dans cette zone, par la réalisation d'excavations, ne peut être occulté.

Les contraintes de l'exploitation du sable en zone continentale ne peuvent autoriser en aucun cas une permissivité quelconque dans l'exploitation de gisements marins sans aucune étude de faisabilité préalable et une étude d’impact sur l'environnement validée. Il s'agit, loin de la recherche du profit, de contribuer à une meilleure compréhension des enjeux se rapportant à l'un des aspects majeurs du développement durable de la zone littorale du Maroc. 
La connaissance de la Mer :

un vecteur du développement durable en Méditerranée

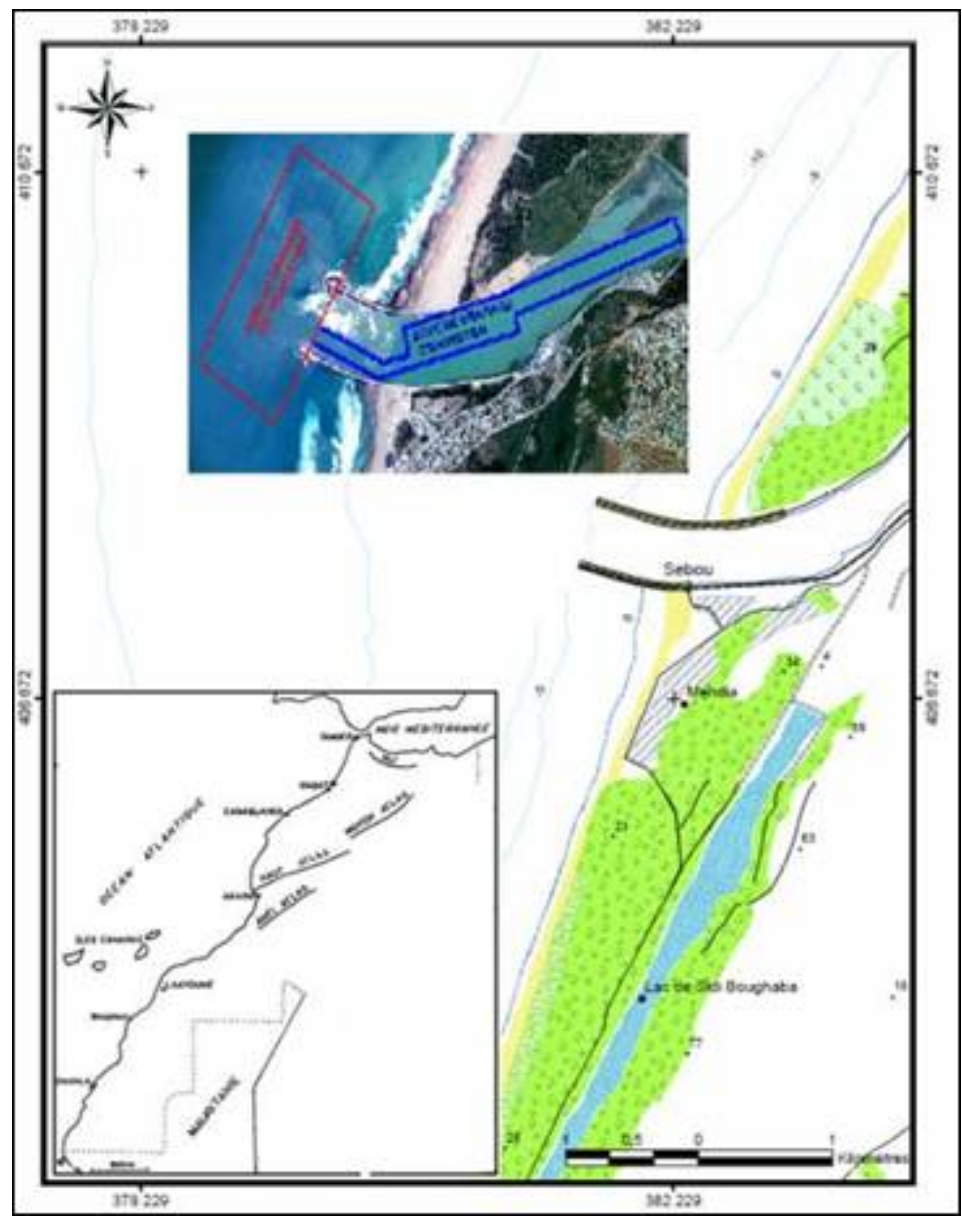

Figure 1 Situation de la zone d'étude et localisation des zones de dragage.

\section{Références bibliographiques}

COMMISSION EUROPEENNE (2011). La mise en œuvre d'activités extractive non énergétiques conformément aux critères Natura 2000. Document d'orientation de la Commission Européenne, juillet 2010, Office des publications de l’Union européenne, Luxembourg, 158 p.

CLARK J. (1977). Coastal Ecosystems Management. New York, John Wiley.

EL ABDELLAOUI J., OZER A. (2005). Apports des images satellitaires à haute résolution spatiale pour l'étude des cordons d'avant-côte sur le littoral atlantique de Tanger (Maroc). Télédétection, Vol. 5, n (1-2-3), pp 81-94.

KOMAR P.D. (1998). Beach processes and sedimentation. Prentice Hall. Upper Saddle River, New Jersey, 544 p.

RBOUB A. (2011). Sable de dragage: un rapport scientifique accablant. L’Economiste n 3499, Casablanca, pp 4-6.

SOGREAH (2011). Etude d'expertise sédimentologique de la plage de Mehdia. Rapport final $\mathrm{N}^{\circ} 1712781$. 